\title{
Die banlieues als Gegenorte der République \\ Eine Diskursanalyse neuer Sicherheitspolitiken in den Vorstädten Frankreichs
}

\section{Mélina Germes, Georg Glasze, Erlangen}

\section{Einleitung: Die banlieues als Objekt neuer Sicherheitspolitiken}

«L'Etat républicain doit être le garant de la paix publique, et j'entends que cette paix soit assurée partout, dans les banlieues comme ailleurs.»

«Der republikanische Staat muss der Garant für den öffentlichen Frieden sein, und ich verlange, dass dieser Frieden überall gewährleistet wird, in den banlieues genau so wie überall sonst.»

Quelle: FrançoIs MitTERAND, Staatspräsident, 11.06.1991 $(\text { Zitat } 1)^{\mathrm{i}}$

Die banlieues werden in dem vorangestellten Zitat als ein besonderes Ziel staatlicher Sicherheitspolitiken hervorgehoben. Tatsächlich lässt sich zeigen, dass insbesondere in den Grosswohnsiedlungen (den so genannten cités) der banlieues in den letzten Jahren in Frankreich spezifische neue Sicherheitspolitiken etabliert wurden (Bonelli 2003). Im Folgenden stellen wir dar, wie im Rahmen der Debatten um Innere Sicherheit in der französischen Politik sowie von Praktikern neuer Sicherheitspolitiken die banlieues als gefährliche Orte konstitutiert werden, welche «die Republik» herausfordern.

In den Sozialwissenschaften finden sich zahlreiche Arbeiten, die sich dem Themenfeld der «banlieues» widmen. Dabei lassen sich zunächst Arbeiten identifizieren, welche die banlieues als Raumcontainer fassen, d.h. als die Vorstädte ausserhalb der Innenstädte, und welche auf der Basis statistischer Daten die Entwicklung dieser Räume darstellen (z.B. Wintol de Wenden 2007). Mehrere Arbeiten haben die Präsentation der banlieues in den Medien untersucht (z.B. Bachmann \& BASIER 1989; Berthaut 2008; KaLInIC 2008). Auf die gesellschaftliche Funktion der Konstitution von banlieues als Problemorte zielen die Arbeiten von Tissot und Poupeau (2005), Estèbe (2004), Tissot (2007) sowie die Arbeiten von Dikeç $(2006,2007)$ ab. Diese Autoren argumentieren, dass die französische Stadtpolitik (politique de la ville), die eng mit den Grosswohnsiedlungen in den Vorstädten verknüpft ist und daher vielfach als politische Antwort auf die «Krise der banlieues» interpretiert wird, letztlich soziale Probleme verräumlicht, indem beispielsweise der wirtschaftliche Strukturwandel oder eine Zuwanderungsgesellschaft als Probleme bestimmter Orte artikuliert und damit als abgegrenzt und bearbeitbar gefasst werden. DIKEÇ interpretiert die banlieues dabei als «badlands of the Republic», als Orte, welche die Integrität der Republik gefährden und gleichzeitig die Etablierung eines «republican penal state» legitimieren (2007: 172ff). An diese Arbeiten schliesst der vorliegende Beitrag an.

In einer diachron angelegten Diskursanalyse der Medienberichterstattung und der Stadtpolitiken in Frankreich haben wir im Rahmen eines anderen Projektes bereits herausgearbeitet, wie die «Krise der banlieues» in den 1980er Jahren zunächst als eine städtebauliche und soziale Krise konstituiert und entsprechende politische Reaktionen entworfen wurden, wie dann seit den 1990er Jahren die Krise zunehmend als ein Sicherheitsproblem gefasst wird und neue spezifische Sicherheitspolitiken für die banlieues entworfen werden und dann, seit Ende der 1990er Jahre, eine zunehmende Verknüpfung zwischen banlieues, Unsicherheit und Fremdheit bzw. ethnischer und religiöser Differenz erfolgt (erste Ergebnisse in Glasze, Germes \& Weber 2009). Damit kann man von einer «Versicherheitlichung» (siehe dazu WÆVER \& BUZAN 2007 sowie Hagmann 2010, in diesem Themenheft) und in jüngster Zeit zudem von einer Ethnisierung des banlieues-Diskurses sprechen.

Dieser Beitrag fokussiert auf die Periode seit Ende der 1990er Jahre und zielt darauf ab, die gesellschaftliche Funktion der Versicherheitlichung des banlieues-Diskurses zu verstehen. Auf der Basis der Hegemonie- und Diskurstheorie von LaClau und Mouffe (1985) entwickeln wir ein Konzept von «Gegenorten» und untersuchen vor dem Hintergrund dieser theoretischen Folie die Reproduktion einer Wir-Identität im Rahmen der Versicherheitlichung des banlieues-Diskurses. Indem die banlieues dabei als Orte des Bedrohlichen und Fremden gefasst werden, wird ein Antagonismus zwischen République und banlieues hergestellt. Auf diese

\footnotetext{
i) Alle Zitate wurden von den Autoren übersetzt. Da wir uns der Problematik der Übersetzung bewusst sind (siehe dazu Husseini 2009), haben wir nach Möglichkeit auch jeweils den Originaltext im Französischen aufgeführt. Die folgenden Zitate haben wir als Beispiele im Rahmen der qualitativen Analyse ausgewählt. Sie zeigen in pointierter Intensität und Deutlichkeit Artikulationsmuster, die regelmässig in den Korpora auftreten.
} 
Weise werden die Werte und Normen und damit die Identität der République reproduziert. Die banlieues werden dabei als «jenseits» der République gefasst, auf paradoxe Weise jedoch gleichzeitig als Teil von ihr - als «verlorene Territorien», die es zurückzuerobern gilt.

\section{Konzeption und Operationalisierung}

\subsection{Identität und Raum: Vorschlag zur Konzeption von «Gegenorten»}

Wir schlagen im Folgenden das Konzept «Gegenorte» vor, das uns geeignet scheint, die Rolle von räumlichen Differenzierungen bei der Herstellung von Identitäten $\mathrm{zu}$ verstehen. Ausgangspunkt dieser Überlegungen sind zwei kurze Vorträge von Michel Foucault, in denen er über Existenz und Funktion von «Gegenräumen» - contre espaces - und «anderen Orten» - hétérotopies - nachdenkt (Foucault 2005 [1984]). Er diskutiert die Bedeutung marginaler und verbotener Räume - d.h. die soziale Funktion von Räumen, die von «normalen» bzw. «neutralen» Räumen abgegrenzt werden (2005 [1984]: 10). Foucault hat diese frühe Idee konzeptionell nicht weiter entwickelt und erst kurz vor seinem Tod der Veröffentlichung der Manuskripte zugestimmt. Problematisch an der Rezeption des Heterotopie-Konzeptes ist, dass vielfach versucht wurde, Heterotopien als bestimmte, abgrenzbare Orte zu fassen (Marchart 1998). Das Problem einer solchen Konzeption scheint zu sein, dass letztlich jeder Ort mit spezifischen Normen verknüpft wird, und damit jeder Ort als Heterotopie im Sinne Foucaults bezeichnet werden kann. Es fehlt augenscheinlich eine breitere gesellschaftstheoretische Einbettung der Idee FoucAults.

Aufbauend auf frühen diskurstheoretischen Arbeiten Foucaults $(1966,1969)$ hat Edward SaId $(1978)$ gezeigt, dass der «Orientalismus» als ein hegemonialer, eurozentrischer Diskurs verstanden werden kann, der eine Region «Orient» schafft, diese als rückständig beschreibt und damit die koloniale Eroberung und Verwaltung legitimiert. Es war insbesondere GREGORY, der die Arbeiten des Literatur- und Kulturwissenschaftlers SAID für die Humangeographie erschlossen hat $(1994,1995,2004)$ und damit eine rege und fruchtbare Diskussion darüber angeschoben hat, inwiefern Identitätskonstruktionen des Eigenen immer wieder auch auf räumliche Differenzierungen zwischen Räumen des Eigenen und Räumen des Anderen zurückgreifen.

Die Hegemonie- und Diskurstheorie, wie sie seit 1985 von Ernesto Laclau und Chantal Mouffe entwikkelt wird, baut auf den Arbeiten von Michel FoucaulT auf, integriert aber darüber hinausgehend auch hegemonietheoretische Überlegungen in der Nachfolge von Antonio Gramsci, dezidiert poststrukturalistische Ansätze, insbesondere von JACQues Derrida, sowie die Konzeptionalisierung von Prozessen der Identitätsbildung bei JaCQues LaCAN. Die Diskurs- und Hegemonietheorie bietet auf dieser Basis eine konsistente theoretische Folie, welche die Zusammenhänge zwischen einer diskursiven Konstitution von Identitäten und gesellschaftlichen Hegemonien darstellt, dabei aber gleichzeitig deren Brüchigkeit und Veränderlichkeit in den Blick zu nehmen vermag. Über die von SAID und GREgory angestossene Diskussion hinausgehend bietet die Diskurs- und Hegemonietheorie daher eine Chance, die Zusammenhänge zwischen der Herstellung von Räumen und der (Re-) Produktion von Identitäten und damit von Gesellschaft zu konzeptionalisieren und dabei auch die Veränderlichkeit und Brüchigkeit von Räumen und Identitäten herauszuarbeiten (Glasze 2009; Glasze \& Mattissek 2009).

Kernidee von Laclau und Mouffe ist, dass kollektive Identitäten und damit Gemeinschaften nicht auf einen wesenhaften gemeinsamen Ursprung zurückgeführt werden können. Gemeinschaftsbildung sei kein «naturwüchsiger Prozess». Vielmehr sei es prinzipiell offen, um welche Knotenpunkte Äquivalenzketten zwischen ansonsten differenten Elementen aufgespannt werden und auf diese Weise eine Gemeinschaft konstituiert wird. Dieser als signifikant zu verstehende Knotenpunkt bricht die Logik der Differenz und ermöglicht so die Logik der Äquivalenz zwischen den Elementen, d.h. Mitgliedern einer Gemeinschaft (Laclau \& Mouffe 1985).

In Anlehnung an Derrida betonen Laclau und Mouffe die Rolle des «konstitutiven Aussen» für die Identitätsbildung. Gerade weil es keine wesenhaften, positiven Identitäten gebe, sei die Abgrenzung vom Aussen entscheidend für die Etablierung von Identitäten. Die gemeinsame Identität werde also durch den gemeinsamen Antagonismus gegenüber dem Aussen hergestellt. LACLAU veranschaulicht dieses Argument mit der Artikulation, d.h. Verknüpfung und In-Beziehung-Setzung verschiedenster Regionen von der nordafrikanischen Atlantikküste über die Arabische Halbinsel bis nach Ostasien als eine einzige Weltregion um den Knotenpunkt «Orient». Diese Konstitution wird nur möglich, weil «Orient» dabei gleichbedeutend mit der Abgrenzung gegenüber «dem Westen» ist: «Orient» ist also gleich «Nicht-Westen» (LacLAU 1990: 32). Das Aussen blockiere allerdings auch die vollkommene Ausbildung einer Identität, indem es die Kontingenz jeglicher Identität zeige (ebd. S. 21). ŽıžEK (1998 [1990]) weist unter Bezug auf LACAN darauf hin, dass jede Identität bereits «in sich selbst blockiert» sei - eine vollkommene, geschlossene und stimmige Identität bleibe immer nur Ziel und werde niemals erreicht. Die «intrinsische, immanente Unmöglich- 


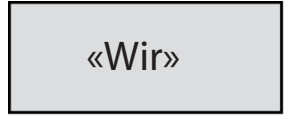

III

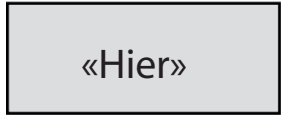

$\neq$

Legende:

||| ̈̈quivalenz

$\neq$ Antagonismus

Abb. 1: Konzeptualisierung des Gegenortprinzips (Antagonismus und Äquivalenzsetzungen)

Conceptualisation of the principle of counterplaces (logics of antagonism and equivalence)

Conceptualisation du principe de contre-lieu (antagonisme et équivalence)

Quelle: eigene Darstellung

keit» von Identität werde also auf ein Aussen, einen antagonistischen Gegner, «projiziert» (S. 126).

Unser Vorschlag ist, in Anlehnung an Laclau und Mouffe, die diskursiv konstitutierten Orte des Anderen als «Gegenorte» zu fassen. Indirekt wird mit der Differenzierung zwischen einem «hier» und «dort» also eine Differenzierung zwischen einem «wir» und den «anderen» hergestellt (s. Abb. 1). Die räumliche Differenzierung wird als eine diskursive Technik gefasst, die dazu dient, die Anderen implizit herzustellen.

Wir gehen davon aus, dass die banlieues im Rahmen der Versicherheitlichung des banlieues-Diskurses als Gegenorte, als «dort», artikuliert werden und untersuchen die Elemente dieser Artikulation: Was ist das «wir» und das «hier» in diesem Sicherheitsdiskurs - was sind die anderen?

\subsection{Forschungsdesign: Untersuchung zweier machtvoller Sprecherpositionen}

Wir konzentrieren uns auf die Untersuchung von Sprecherpositionen, die diskursiv legitimiert sind, im Namen eines «wir» der Gesellschaft zu sprechen. Dazu haben wir zwei Textkorpora zusammengestellt: Zum einen haben wir einen Textkorpus mit Reden von Staatspräsidenten und Ministern zu Themen der Inneren Sicherheit zusammengestellt (Korpus I; 19902008), und zum anderen haben wir 20 Interviews mit Vertretern nationaler und lokaler Institutionen der Sicherheitspolitik in Paris sowie den Pariser banlieues geführt (2008-2009) und die Interviewtranskripte in einem zweiten Korpus (II) zusammengeführt. Auf der Ebene des Zentralstaats wurden Interviews im Innenministerium und dem interministeriellen Generalsekretariat für Prävention (Secrétariat Général du Comité Interministériel de Prévention de la Délinquance) sowie dem Komitee für die Umsetzung der französischen Stadtpolitik (politique de la ville) geführt (Comité Interministériel des Villes). Auf der Ebene des département und auf der kommunalen Ebene wurde zum einen ein Vertreter der Polizeidirektion im nördlich von Paris gelegenen département Seine-Saint-Denis (93) interviewt sowie Vertreter der kommunalen Sicherheitsämter und der Stadt-, Sozialund Wohnungsämter in fünf Gemeinden (Bobigny, Montfermeil, Saint-Denis, Aulnay, Clichy-sous-Bois) in diesem département. Das département Seine-SaintDenis (93) eignet sich als lokale Fallstudie, da das «93» in Medien und Politik in Frankreich regelmässig als das département beschrieben wird, in dem sich die Probleme der banlieues konzentrieren (z.B. städtebaulich problematische Grosswohnsiedlungen, hohe Arbeitslosigkeit, hohe Ausländeranteile, hohe Kriminalitätsraten), und das als «Experimentierfeld» und besonderer Gegenstand von politischen Massnahmen gefasst wird.

Diese beiden Korpora untersuchen wir mit aussagenanalytischen Verfahren (zur Einführung siehe MATTISSEK 2009). Verfahren der Aussagenanalyse nehmen die Materialität der Form als Ausgangspunkt, d.h. sie gehen nicht davon aus, dass die Bedeutung von Texten durch einen dahinterliegenden Sinn bestimmt wird, sondern postulieren, dass Sinn einen instabilen Effekt darstellt, der im Zusammenspiel sprachlicher (materialer) Formen und dem jeweiligen interpretativen Kontext immer wieder aufs Neue hervorgebracht wird (ANGERMÜLLER 2007: 104). In den Aussagenanalysen wird zugrunde gelegt, dass die Bedeutung einer Aussage nicht kontextunabhängig bestimmt werden kann, dass die Bedeutungskonstitution aber dennoch bestimmten Regeln folgt. Ziel ist es folglich, die Regeln bzw. Muster der Aussagen offen zu legen.

Die Untersuchung der Textkorpora zielt also darauf $\mathrm{ab}$, regelmässige Verknüpfungen bestimmter sprachlicher Elemente herauszuarbeiten. Zwei methodische Instrumente ermöglichen es dabei, diese Untersuchungen strukturiert und nachvollziehbar zu gestalten. Das Verfahren der «semantischen Strickleiter» basiert auf der Annahme, dass Bedeutungen immer in einem semantischen Verhältnis von Äquivalenzen und Differenzen hergestellt werden. Damit können in einem Text die Äquivalenzen und Differenzen wie in einer Strickleiter herausgearbeitet werden und auf diese Weise auch nicht explizit Ausgesagtes, aber implizit Mitgesagtes abgeleitet werden. Das Verfahren 
der «semantischen Strickleiter» (für eine Anwendung siehe HöHNE 2003) erscheint uns geeignet, das nicht explizit Gesagte, das Implizite, herauszuarbeiten, das mit der (expliziten) Konstitution von Gegenorten einhergeht. Das Konzept der Polyphonie ermöglicht es darüber hinaus, Brüche und Widersprüche innerhalb von Aussagen als unterschiedliche «Stimmen» innerhalb eines Textes zu fassen (DUCROT 1984).

Unsere Untersuchung konzentriert sich damit auf die Artikulationen, die von einflussreichen Sprecherpositionen in Politik und Verwaltung getätigt werden und hegemoniale Diskurse (re-) produzieren. Ausserhalb des Schwerpunkts dieser Untersuchung liegen damit andere teilweise dezidiert gegenhegemoniale Diskurse (Hinweise dazu liefern DiKeÇ 2007: 129ff.; HÜSER 2004; LAPEYRONNIE 2005 und SchumanN 2005).

\section{Die diskursive Konstitution der banlieues als bedrohliche Gegenorte der République}

Auf der Basis einer Analyse von Mediendiskursen konnten wir im Rahmen anderer Studien bereits zeigen, dass die banlieues in der Medienberichterstattung als Problemorte und gefährliche Orte produziert werden: als Orte der Gewalt und des Konflikts mit der Mehrheitsgesellschaft (Brailich et al. 2010; Germes et al. 2010). Im Folgenden wenden wir uns der Frage zu, inwiefern die banlieues im Kontext der Versicherheitlichung des banlieues-Diskurses als Gegenorte artikuliert werden, und welche Identitätskonstruktionen des Eigenen damit einhergehen.

Mithilfe des Instruments der semantischen Strickleiter haben wir zunächst den Korpus mit Reden von Staatspräsidenten und Ministern untersucht. Dabei zeigt sich, dass teilweise explizit, teilweise implizit eine Opposition zwischen der République und ihren Werten und den banlieues konstituiert wird, so dass die Bedeutung von «République» damit (re-) produziert wird (s. Abb. 2 - in Klammer stehen die nicht explizit genannten Begriffe).

Es zeigt sich, dass das «wir» fast durchweg explizit über das Wort République bezeichnet wird, welches mit Begriffen wie «nationale Werte» äquivalent gesetzt wird, während das Andere nur implizit mittels der Nennung von banlieues und quartiers (Viertel) artikuliert wird (s. Abb. 2 ). Die banlieues dienen dabei als konstitutives Aussen für das «wir» der République (s. Abb. 3).

Explizit genannt wird also das «wir» und das «dort»nicht aber «die Anderen». Im Folgenden möchten wir daher die Gestalt des «Anderen», wie sie im Rahmen der Versicherheitlichung des banlieues-Diskurses hergestellt wird, näher untersuchen.

\section{Die «Anderen»: zwei paradoxe Gestalten}

Um die Umrisse der Gestalt des «Anderen» genauer bestimmen zu können, stützen wir uns auf eine Untersuchung von Transkriptionen der Reden von Präsidenten und Ministern sowie von Interviews mit Vertretern kommunaler und departementaler Organisationen und Ämter. Die Umrisse der Gestalt des Anderen lassen sich danach entlang zweier Linien zeichnen: Im Vordergrund steht das Bild des Anderen als bedrohliche jeunes de banlieue (Jugendliche aus der banlieue). Eher im Hintergrund, aber in enger Beziehung zu diesem Bild, spielt die Qualifizierung des Anderen als fremd eine zentrale Rolle. Neben dieser Figur des bedrohlichen und fremden (und jungen) Anderen wird aber auch eine zweite Figur entworfen: die Figur der bedrohten Opfer in den banlieues. Eine Figur die «eigentlich» auf der Seite der République steht.

\subsection{Das Konstrukt des «jeune de banlieue» - fremd, nicht-integriert, männlich und bedrohlich}

Das Wort jeunes (jung, Jugendliche) ist das Wort, das in der Berichterstattung von Le Monde im höchsten Masse charakteristisch für die Umgebung von banlieue ist (Germes et al. 2010), und das regelmässig in zwei spezifischen Artikulationen (jeunes de banlieue, jeunes en difficulté) in den untersuchten Textkorpora auftaucht (siehe Zitate 4, 7,13 und 14). Dies ist nicht zuletzt auf die regelmässige Verwendung der Wortfolge jeunes de banlieue zurückzuführen. Diese Wortfolge verschränkt Jugendliche unmittelbar mit einer raumbezogenen Kategorie (dazu auch Bonelli 2008; Hancock 2008). In einer Art doppelter Korrespondenz laden sich banlieues (auch cités, quartiers) und jeunes gegenseitig mit Bedeutung auf. Jugendliche werden als eine problematische und marginalisierte Gruppe artikuliert, indem sie in Verbindung mit Wörtern wie «Konflikte» oder «Probleme» gestellt oder mit der euphemistischen Umschreibung jeunes en difficulté (Jugend in schwierigen Lebensumständen) artikuliert werden. Die regelmässige Verknüpfung von jeunes de banlieue mit Bedrohung, Unsicherheit und Gewalt (beispielsweise auch durch die Verwendung von Wörtern wie sauvageons - Wilde - und racaille - Abschaum) führt dazu, dass im diskursiven Kontext von banlieue das Wort jeunes letztlich immer als synonym für potentiell delinquent und bedrohlich gelesen wird (siehe dazu bereits die Hinweise von SAUvadET 2006).

Insgesamt werden die quartiers (Stadtviertel) regelmässig mit kultureller Differenz verknüpft (BRAILICH et al. 2008). Dem Polyphonie-Konzept folgend lassen sich dabei zwei Stimmen unterscheiden. Zum einen wird kulturelle Differenz mit Vielfalt und Kreativität und damit als Potential beschrieben, zum anderen, und vielfach im gleichen Atemzug, aber auch als Problem. 


\begin{tabular}{|c|c|c|}
\hline $\begin{array}{c}\text { republikanischer Staat } \\
\text { öffentliche Sicherheit } \\
\text { überall sonst }\end{array}$ & $\begin{array}{l}\longleftrightarrow \text { (andere Ordnung) } \\
\stackrel{\mid}{\longleftrightarrow} \text { (Unsicherheit) } \\
\mid \\
\qquad \text { banlieues }\end{array}$ & $\begin{array}{l}\text { Zitat 1: } \\
\text { "L'Etat républicain doit être le garant de la paix publique, } \\
\text { et j'entends que cette paix soit assurée partout, dans les } \\
\text { banlieues comme ailleurs.» } \\
\text { «Der republikanische Staat muss Garant für den öffentli- } \\
\text { chen Frieden sein, und ich verlange, dass dieser Frieden } \\
\text { überall gewährleistet wird, in den banlieues genau so } \\
\text { wie überall sonst.» }\end{array}$ \\
\hline $\begin{array}{c}\text { Sicherheit } \\
\text { nicht-benachteiligte } \\
\text { Viertel unserer Städte } \\
\text { weniger Widerstände } \\
\text { gegen Integration von } \\
\text { Jugendlichen } \\
\text { (keine Migration) } \\
\text { République }\end{array}$ & $\begin{array}{l}\text { benachteiligte } \\
\text { Viertel }\end{array}$ & $\begin{array}{l}\text { Zitat 2: } \\
\text { «Comment ne pas voir que la sûreté est (...) la plus } \\
\text { menacée dans les quartiers les plus déshérités de nos villes } \\
\text { et de nos banlieues, là où l'intégration des jeunes à la } \\
\text { République, et notamment des jeunes d'origine étrangère, } \\
\text { rencontre le plus d'obstacles?» } \\
\text { «Wie kann man übersehen, dass die Sicherheit (...) am } \\
\text { meisten in den benachteiligten Vierteln unserer Städte } \\
\text { und unserer banlieues gefährdet ist, dort wo die Inte- } \\
\text { gration der Jugendlichen in die République und beson- } \\
\text { ders derjenigen mit Migrationshintergrund auf die } \\
\text { meisten Widerstände trifft?» }\end{array}$ \\
\hline $\begin{array}{c}\text { (Frankreich) } \\
\text { Gesetz der République } \\
\text { nationale Werte } \\
\text { nationale Einheit }\end{array}$ & $\begin{aligned} & \longleftrightarrow \text { unsere banlieues } \\
& \longleftrightarrow \text { Gesetz der Banden } \\
& \longleftrightarrow \text { urbane Ghettos } \\
& \longleftrightarrow \text { eigene Kulturen } \\
& \text { (keine nationalen } \\
& \text { Werte) } \\
& \longleftrightarrow \text { Insel des Kommuni- } \\
& \text { tarismus }\end{aligned}$ & $\begin{array}{l}\text { Zitat 3: } \\
\text { "La réalité de nos banlieues c'est que nous avons } \\
\text { accepté que la loi des bandes s'impose au détriment de } \\
\text { celle de la République (...) que nous avons laissé se } \\
\text { développer de véritables ghettos urbains, (...) qu'ils } \\
\text { vivent des cultures identitaires en marge des valeurs } \\
\text { nationales, ont constitué des îlots de communautarisme } \\
\text { qui portent atteinte à l'unité nationale.» } \\
\text { «Die Wahrheit unserer banlieues ist, dass wir akzeptiert } \\
\text { haben, dass sich das Gesetz der Banden gegenüber dem } \\
\text { der République durchsetzt (...), dass wir die Entwicklung } \\
\text { von urbanen Ghettos zugelassen haben, (...) dass sie in } \\
\text { eigenen Kulturen leben, die die nationalen Werte in } \\
\text { Frage stellen und [dass sie] kleine Inseln des Kommuni- } \\
\text { tarismus geschaffen haben, welche der nationalen Ein- } \\
\text { heit schaden.» }\end{array}$ \\
\hline
\end{tabular}

Abb. 2: Diskursive Auseinandersetzung zwischen République und banlieues Controversial discourse involving the concepts of «République» and «banlieues» Polémique discursive entre République et banlieues Quelle: Korpus I. Eigene Darstellung auf der Basis von Zitat 1 sowie von Reden der vormaligen Innenminister J.-P. Chevènement (Zitat 2 vom 24.10.1997) und N. SArkozy (Zitat 3 vom 28.11.2005)

Interessant ist dabei auch der regelmässige Gebrauch der Metapher vivre ensemble (zusammen leben) durch die Interviewpartner. Damit wird einerseits eine Einheit hergestellt, aber andererseits gleichzeitig die Aufforderung und damit indirekt ein Problem («wir müssen zusammen leben») konstituiert. Es zeigt sich, dass Jugendliche insbesondere auch deswegen als gefährlich gelten, weil sie gleichzeitig als fremd beschrieben werden.

\section{Zitat 4:}

"On a une population qui est particulière, cosmopolite avec ses richesses, ses qualités (...) et ses défauts! Les défauts aussi de la diversité c'est (...) bon, c'est ça, c'est la délinquance.» 
«Wir haben eine besondere und kosmopolitsiche Bevölkerung, mit ihrem Reichtum, mit Qualitäten (...) und Fehlern! Die Probleme der Vielfalt sind auch (...) nun ja, es ist die Kriminalität.»

Zitat 5:

«On ne mettra pas fin durablement aux violences urbaines et à la délinquance quand deux millions de jeunes se sentent trop souvent exclus des mécanismes de l'intégration et de l'ascension sociale. Je pense en particulier aux jeunes d'origine maghrébine.»

«Wir werden die urbane Gewalt und die Kriminalität nicht dauerhaft beenden, wenn zwei Millionen Jugendliche sich zu häufig aus den Prozessen der Integration und des sozialen Aufstiegs ausgeschlossen fühlen. Ich denke dabei besonders an die Jugendlichen maghrebinischer Herkunft.»

Zitat 6:

«De plus en plus de jeunes filles sont menacées et insultées dans leur lieu de vie comme à l'école. Par crainte, beaucoup se cachent et restreignent leur liberté, lui assignant des limites étroites, étrangères à notre culture.»

«Immer mehr Mädchen werden in ihrem Lebensumfeld, so wie in der Schule, bedroht und beleidigt. Aus Angst verstecken sich viele und schränken ihre Freiheit durch enge Grenzen ein, die für unsere Kultur fremd sind.»

Quelle: Korpus II: Interview mit einem Vertreter der Polizei auf der kommunalen Ebene im département Seine-SaintDenis (93) (Zitat 4); Korpus I: Reden des vorm. Innenministers J.-P. ChevÈnEMENT (Zitat 5 vom 18.03.1999) und des vorm. Präsidenten J. ChIRAC (Zitat 6 vom 19.02.2002)
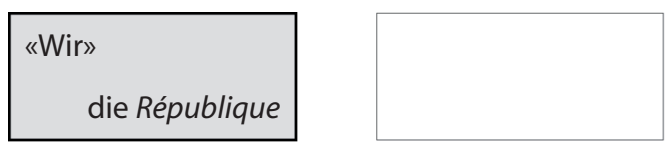

$\neq$
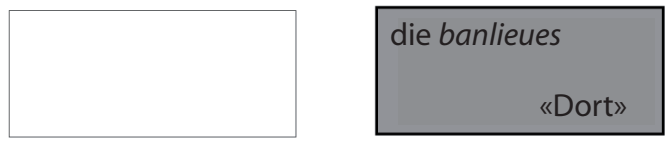

Legende:

$\neq$ Herstellung eines Antagonismus

Abb. 3: Erster Schritt des Gegenortprinzips: Bezeichnung von «Wir» und «Dort»

First stage of the principle of counterplaces: reference to «us» and «there»

Première étape du principe de contre-lieu: désignation $d u$ «Nous» et $d u$ «Ici»

Quelle: eigene Darstellung
In zahlreichen Aussagen (z.B. Zitat 4) wird eine Beziehung zwischen einer fremden Herkunft, einer mangelnden Integration in die französische Gesellschaft und einer damit ursächlich zusammenhängenden Gewalt artikuliert (JABLONKA 2008). Allerdings werden in den Reden und Interviews niemals explizit Fremdheit und Gefahr äquivalent gesetzt - die Relationierung erfolgt immer über Zwischenschritte (Einwanderung, fehlende Integration, problematische Jugendliche).

Der Unsicherheitsdiskurs greift zudem eine Differenzierung entlang des sozialen Geschlechts auf (CoUTRAS 2003): Frauen werden als Opfer, Männer als Täter artikuliert. Am Beispiel der Frauenrolle wird eine Grenzlinie zwischen einer Kultur des Eigenen und einer Kultur des Anderen gezogen (Zitat 6). Die fremde Kultur wird mit Beschränkungen und Gewaltakten gegenüber Frauen verknüpft. Die Opferrolle der Frauen wird mit kultureller Fremdheit verknüpft und auf diese Weise indirekt die Kategorie der bedrohlichen, männlichen fremden (und jugendlichen) Anderen hergestellt (Abb. 4).

\subsection{Die diskursive Aufspaltung der Anderen in Bedrohende und Bedrohte}

Das Beispiel der Frauen in der (zu schützenden) Opferrolle deutet bereits an, dass zwei Andere hergestellt werden. Zahlreiche Aussagen aus der Sprecherposition des «wir» beschäftigen sich mit einer Grenzziehung zwischen den «würdigen Anderen» und den «feindseligen Anderen» (siehe Abb. 5).

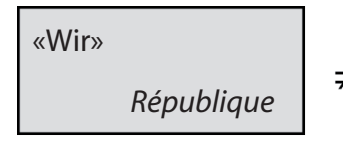

III

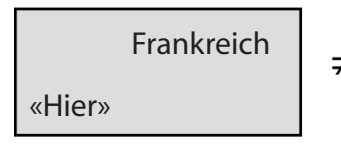

Legende:

III Äquivalenz

$\neq$ Antagonismus
Abb. 4: Zweiter Schritt des Gegenortprinzips: Bezeichnung der «Anderen», die zum «Dort» gehören

Second stage of the principle of counterplaces: reference to «others» that belong to «there»

Deuxième étape du principe de contre-lieu: désignation des «Autres» appartenant à l' «Ici»

Quelle: eigene Darstellung 


\begin{tabular}{|c|c|}
\hline $\begin{array}{cl}\text { Bürger } & \text { Delinquenten } \\
\mid & \mid \\
\text { sollen [von der } & \begin{array}{l}\mid \\
\text { ihnen soll [von der Polizei] } \\
\text { Polizei] be- } \\
\text { das Leben schwer gemacht } \\
\text { werden }\end{array}\end{array}$ & $\begin{array}{l}\text { Zitat 7: } \\
\text { «Il faut que la police puisse rentrer de façon complètement } \\
\text { aléatoire dans les quartiers pour compliquer la vie des } \\
\text { délinquants et rassurer le citoyen qui lui n'aspire qu'à une } \\
\text { seule chose, c'est vivre en toute tranquillité.» } \\
\text { «Die Polizei muss nach dem Zufallsprinzip in die Viertel } \\
\text { eindringen können, um den Delinquenten das Leben } \\
\text { schwer zu machen und den Bürger zu bestärken, der nur } \\
\text { nach einer einzigen Sache strebt, nämlich nach einem } \\
\text { ungestörten Leben.» }\end{array}$ \\
\hline $\begin{array}{l}\text { an uns heran- } \longrightarrow \text { von uns isolieren } \\
\text { rücken }\end{array}$ & $\begin{array}{l}\text { Zitat 8: } \\
\text { «Il faut faire le tri entre les uns et les autres. Il y a une } \\
\text { minorité à isoler, et une majorité à rapprocher de nous.» } \\
\text { «Man muss die Einen von den Anderen trennen. Es gibt } \\
\text { eine Minderheit, die wir von uns isolieren und eine Mehr- } \\
\text { heit, die wir an uns heranrücken müssen.» }\end{array}$ \\
\hline
\end{tabular}

Abb. 5: Diskursive Aufspaltung der «Anderen» in Opfer und Täter Discursive differentiation of the «others» in victims and perpetrators Division discursive des «Autres» en victimes et agresseurs Quelle: eigene Darstellung am Beispiel einer Rede von N. SARKOZY, vorm. Innenminister, am 4.5.2006 (Korpus I) und eines Interviews mit einem Vertreter des Sicherheitsamtes auf kommunaler Ebene (Korpus II)

Damit wird eine Grenze zwischen einer Minderheit (der feindseligen, nicht aufrichtigen Täter) und einer Mehrheit (integrationswillig, mit guten Werten und ggf. Opfer) gezogen. Die zweite Gruppe wird als würdig beschrieben, die Werte der République anzunehmen, sich $\mathrm{zu}$ integrieren, aufgenommen $\mathrm{zu}$ werden. Die Differenzierung stützt und legitimiert die Wir-Identität und leitet die Praxis beispielsweise der Polizei an: Heranführen der Integrationswilligen und Isolation der Feindseligen (Abb. 6).

\section{Die Wiederaneignung der verlorenen Territorien durch die République}

Der letzte Schritt unserer Analyse fokussiert auf die neuen Sicherheitspolitiken vor Ort und basiert auf den Interviews mit Vertretern von Organisationen der Sicherheitspolitik in einigen Vororten im Norden von Paris. In der Analyse lässt sich wiederum die Konstitution eines «wir» der République rekonstruieren, das drei Facetten hat.

\subsection{Konflikt über verlorene Territorien}

Die Interviewpartner in den Ordnungs- und Sicherheitsämtern der Kommunen verorten Kriminalität und Gefahr vielfach explizit in Grosswohnsiedlungen. Die Auseinandersetzung mit Unsicherheit und Kriminalität wird als territorialer Konflikt artikuliert. Manche Grosswohn- siedlungen werden zudem als no go areas beschrieben, in denen kein Polizist geduldet wird, in denen andere Gesetze und andere Normen gelten (s. Zitate).

\section{Zitat 9:}

«Il y a un quartier qui est plus tout à fait sous les lois de la République, qui est tenu par un caïd. Donc là vous reposez le problème de la mafia, (...) j'exagère pas.»

«Es gibt ein Viertel, das nicht mehr ganz unter dem Gesetz der République steht und von einem Bandenchef beherrscht wird. Dort also liegt das Problem der Mafia, (...) ich übertreibe nicht.»

Zitat 10:

«Des leaders délinquants qui font un peu régner leur loi, et quand ils voient des véhicules de police, qui font tout pour les discréditer, les policiers, qui font tout pour les chasser du territoire. Ils ont une logique de territoire, c'est chez eux, et puis les policiers, ben, c'est en dehors des Bosquets.»

«Bandenführer lassen ihre Gesetze walten, und wenn sie Polizeiautos sehen, tun sie alles, um sie in Verruf zu bringen - also die Polizisten; sie tun alles, um sie von ihrem Territorium zu verjagen. Sie besitzen einen ausgeprägten Sinn für ihr Territorium, das ist schliesslich ihr Gebiet - und die Polizisten, nun ja, die sind ausserhalb [der Grosswohnsiedlung] Les Bosquets.»

Quelle: Korpus II: Interviews mit Sicherheitsbeamten auf kommunaler Ebene 


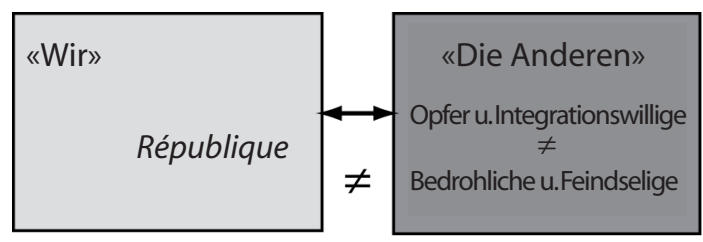

III

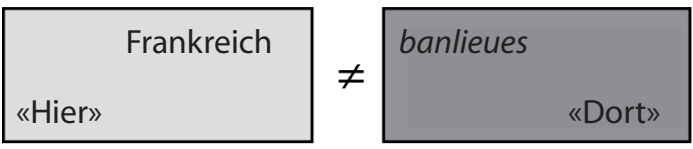

Legende:

III Äquivalenz

$\neq$ Antagonimsus

$\longleftrightarrow$ Bruch des Antagonismus

Abb. 6: Die Aufspaltung der «Anderen»

Differentiation of the concept of «others»

Différenciation des «Autres»

Quelle: eigene Darstellung

Die Grosswohnsiedlungen der banlieues werden regelmässig als «verlorene Territorien» (territoires perdus) der République bezeichnet. Implizit wird damit auch kommuniziert, dass sie eigentlich zum «hier» der République gehören sollten und damit also zurückerobert werden müssen.

Wir interpretieren den Diskurs des «wir» als einen Wiederaneignungdiskurs, indem das «wir»- d.h. die République - als legitime Instanz der Macht verankert wird. Im Folgenden stellen wir dar, inwiefern im Rahmen der neuen Sicherheitspolitiken, die auf Ansätze aus dem Bereich der Sozialpolitik, der Städtebaupolitik, aber auch der Inneren Sicherheit bzw. Polizeiarbeit zurückgreifen, dem «wir» eine Identität gegeben wird, die drei Facetten hat: erstens, die paternalistisch-moralische Autorität, welche «normales» Verhalten definiert, zweitens, der überwachende Eigentümer, der Grosswohnsiedlungen renoviert, umzäunt und kontrolliert sowie drittens, die rückerobernde Gewalt, die die verlorenen Territorien sichert und auf diese Weise für die République zurückgewinnt.

\section{2 «Wir» als moralische Autorität}

Der sozialpolitische Flügel der Präventionspolitiken (prévention de la délinquance) umfasst Massnahmen, die auf Gruppen zielen, die als potentiell gefährdet oder potentiell gefährlich gelten. Dies sind einerseits die Mütter (i.d.R. ausländischer Herkunft) sowie Frauen im Allgemeinen, und andererseits die männlichen Jugendlichen (FAYMAn 1995), die als «Wilde» (sauvageons) beschrieben werden, die es zu zivilisieren gelte (s. Zitate).
Zitat 11:

«Pour les 16-18 ans ça va être un chantier peinture qu'on a monté. On a souhaité avec des jeunes qui sont en rupture de repères, qui sont en grande difficulté, faire un chantier. C'est pas évident d'avoir des ordres de quelqu'un. C'est tout un réapprentissage des règles.»

«Für die 16- bis 18-Jährigen haben wir eine Renovierungsarbeit organisiert. Wir wollten eine Renovierungsarbeit mit den Jugendlichen machen, die unter Orientierungslosigkeit leiden, die in grossen Schwierigkeiten stecken. Es ist gar nicht so einfach, von jemandem Befehle entgegenzunehmen. Das ist eigentlich ein Wiedererlernen von Regeln.»

Zitat 12:

"Il y a l'apprentissage des règles, de l'autorité, de solidarité aussi parce que c'est ça le sport. (...) C'est une structure [le centre loisir jeunesse de la police nationale] qui est gérée par des policiers. Donc ils sont en uniforme. Ils font des activités diverses et variées mais c'est géré par une autorité.»

«Das Erlernen von Regeln, von Autorität, von Solidarität - denn das macht ja den Sport aus. (...) Es ist eine Institution [das Freizeitzentrum für Jugendliche der Nationalpolizei], die von Polizisten geführt wird. Deshalb tragen sie auch Uniform. Es gibt viele und verschiedene Aktivitäten - aber sie wird durch eine Autorität geleitet».

Quelle: Korpus II: Interviews mit Vertretern von kommunalen Sozialämtern

Die Massnahmen zielen darauf, die männlichen Jugendlichen auch ausserhalb der Schulzeit einer Betreuung durch Erzieher (z.T. Polizisten) zu unterwerfen, um ihnen damit Werte und Verhaltensformen zu vermitteln, die sie nicht woanders gelernt haben (z.B. Pünktlichkeit, Autorität, Regeln, Respekt) - Werte und Verhaltensformen, die vom «wir» ausgehen. Das «wir» wird damit als paternalistischer Erzieher, als moralische Autorität hergestellt, dessen Ziel es ist, eine Bevölkerung zu gewinnen, die aufgrund eigener bzw. fehlender Werte bedrohlich und bedroht ist.

\section{3 «Wir» als überwachender Eigentümer}

Die Grosswohnsiedlungen der banlieues sind Ziel von Politiken der städtebaulichen Erneuerung, wobei (auch) die Verbesserung der «Sicherheit» erreicht werden soll. Mit der städtebaulichen Neugestaltung sollen im Sinne der Ansätze des defensible space Räume geschaffen werden, die «sichere» Verhaltensformen begünstigen und deviante Verhaltensformen verhindern (wie Romieux 2007 erklärt). So werden beispielsweise bei den höchsten Gebäuden die obersten Stockwerke abgetragen und die Freiflächen differenziert in dezidiert offen zugängliche und abgeschlossene Räume. Es wird darauf geachtet, dass alle Wege den Fahrzeugen der Polizei offen stehen, und dass Hindernisse für eine gute Einsehbarkeit entfernt 
werden. Darüber hinaus werden Systeme der Videoüberwachung ausgebaut (s. Zitat 13).

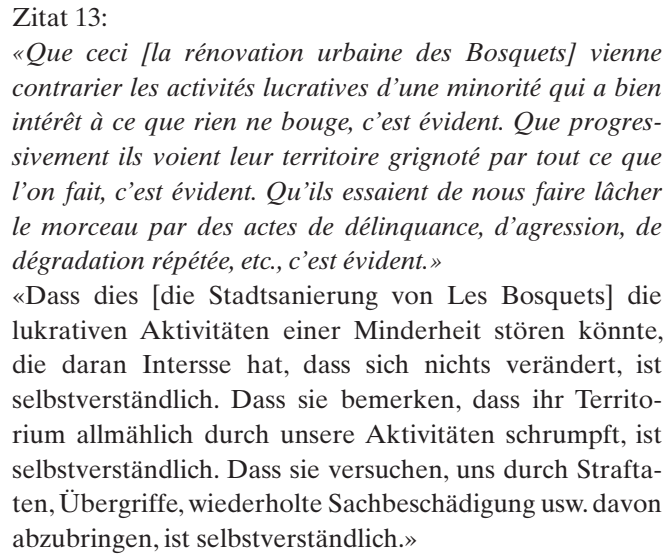

Quelle: Korpus II: Interview mit einem Bürgermeister

Die Stadterneuerung bzw. Sanierung wird beschrieben als eine Wiederaneignung eines Raumes, der bis dahin den Banden gehört hat - eine Wiederaneignung durch das «wir» der République.

\section{4 «Wir» als rüickerobernde Gewalt}

Neben den Sozial- und Städtebaupolitiken, die Sicherheit zu ihrem Ziel machen, werden auch neue Sicherheitspolitiken im engeren Sinne etabliert, die auf die staatlichen Sicherheitskräfte zurückgreifen. Seit Mitte der 1990er Jahre sind neue Sicherheitspolitiken etabliert worden, die spezifisch abgegrenzt in einigen Grosswohnsiedlungen der banlieues greifen (Rigouste 2008).

\section{Zitat 14:}

«La population [de la cité] ces dernières années voyait bien que c'étaient les caïds qui avaient le haut du pavé et qu'il fallait faire profil bas (...) Une population qui depuis la création des UTEQ commence à rebasculer du côté des institutions.»

«Die Bevölkerung [der Grosswohnsiedlung] konnte während der letzten Jahre feststellen, dass die Bandenführer die Macht besassen, und dass man sich unauffällig verhalten musste (...) Diese Bevölkerung beginnt seit der Schaffung der UTEQ, auf die Seite der [staatlichen] Institutionen zurückzukehren.»

\section{Zitat 15:}

"Dix fonctionnaires à pied qui patrouillent dans un secteur, qui prennent des contacts, qui vont interpeller, qui vont contrôler, qui vont sécuriser, qui vont pacifier, qui vont prévenir. On a un car donc qui est autour et prêt à soutenir les fonctionnaires s'ils étaient en difficulté. Le premier jour de la mise en place de l'UTEQ on a eu deux fonctionnai- res blessés. Il fallait que la police arrive à s'imposer dans le secteur pour que la population puisse mieux vivre tout simplement.»

«Zehn Polizisten patroullieren in einem kleinen Bezirk, knüpfen Kontakte, überprüfen Personalien, kontrollieren, sorgen für Sicherheit, greifen ein und leisten Präventionsarbeit. Wir haben einen Polizeibus in der Nähe, der bereitgestellt ist, die Beamten zu untersützen, wenn sie in Schwierigkeiten geraten. Am ersten Einsatztag der UTEQ hatten wir zwei verletzte Polizisten. Es war notwendig, dass die Polizei es schafft, sich in dem Bezirk durchzusetzen, damit die Bevölkerung besser leben kann - ganz einfach.»

Quelle: Korpus II: Interviews mit einem Bürgermeister und einem Vertreter des kommunalen Sicherheitsamtes

Ein Beispiel für die Etablierung spezifischer Sicherheitspolitiken sind die UTEQ (Unités Territoriales de Quartier). Diese werden in einer Experimentierphase seit Mitte 2008 in verschiedenen Grosswohnsiedlungen (7000 Einwohner in der Grosswohnsiedlung in Zitat 15) im département Seine-Saint-Denis eingesetzt. Die UTEQ sind eine bürgernahe, aber repressive Sonderpolizei, die sich jeweils aus zehn bzw. mehr bewaffneten und speziell trainierten Polizisten pro Quartier zusammensetzt. Sie werden im Krisenfall unterstützt von den Compagnies de Sécurisation - einer Art Einsatzpolizei auf der Ebene des département.

Die repressiven Sicherheitspolitiken und die «Rückeroberung» werden im Namen der Opfer-Bevölkerung gerechtfertigt: so wird Gewalt als Massnahme zur Verminderung von Unsicherheit legitimiert. Dieser Diskurs konstituiert das «wir» als einen Kämpfer für die Sicherheit, der mit legitimer Gewalt auf eine illegitime Gewalt und die damit verbundene «Unsicherheit» antwortet. Das Ziel dieser Massnahmen ist, die «verlorenen Territorien» zurückzugewinnen und sie an die Gesetze, Normen und Werte der République heranzuführen.

\section{Fazit}

Im Kontext der Versicherheitlichung des banlieuesDiskurses werden die Vorstädte als Gegenort gezeichnet, an dem andere Werte gelten, und der insgesamt eine Gefahr für die Ordnung und Werte des «wir» darstellt. Gleichzeitig werden damit diese Werte definiert und eine Wir-Identität der République (re-) produziert. Die banlieues sind in diesem Sinne ein konstitutives Aussen der République.

Auf paradoxe Weise werden die banlieues dabei als «verlorene Territorien» konstituiert, die «eigentlich» zur République gehören und deshalb zurückerobert 


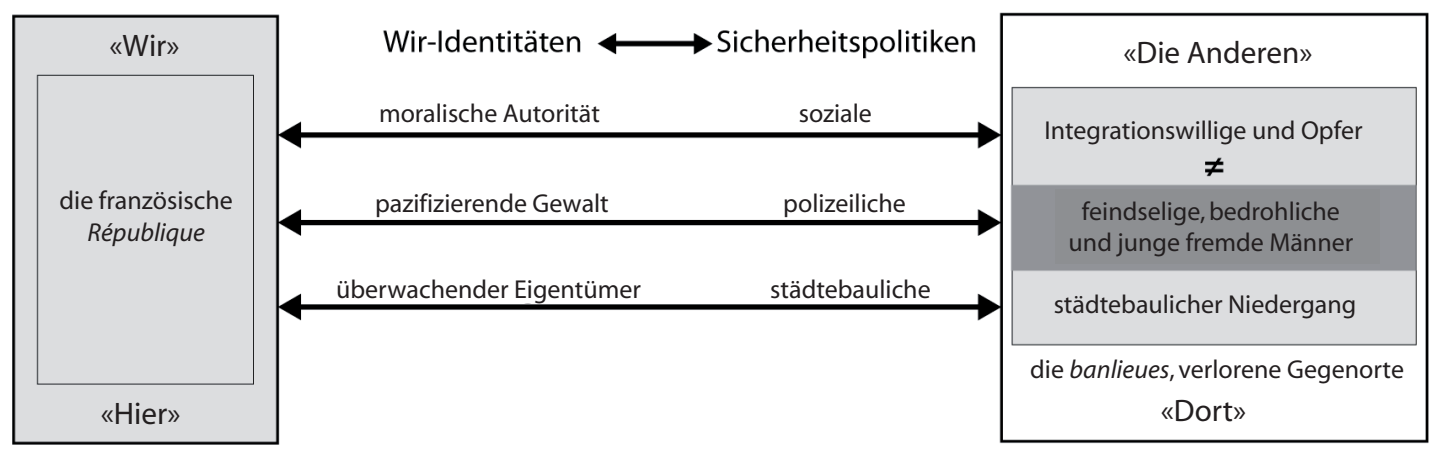

Legende:

$\neq$ Antagonimsus

$\longleftrightarrow$ Bruch des Antagonismus

Abb. 7: Die «Wiederaneignung eines Gegenortes»: Sicherheitspolitiken und Identitätskonstitution

Reintegration of a counterplace: security policy and constitution of identity

La «réappropriation d'un contre-lieu»: politiques de sécurité et constitution de l'identité

Quelle: eigene Darstellung

werden müssen. Die Wiederaneignung wird durch eine Differenzierung der Anderen legitimiert: die gefährlichen Anderen müssen durch die République zurückgedrängt werden, um die gefährdeten Anderen integrieren zu können (Abb. 7).

\section{Literatur}

ANGermülleR, J. (2007): Was fordert die Hegemonietheorie? Zu den Möglichkeiten und Grenzen ihrer methodischen Umsetzung. - In: NonHoff, M. (Hrsg.): Diskurs, radikale Demokratie, Hegemonie. Zum politischen Denken von Ernesto Laclau und Chantal Mouffe. - Bielefeld: transcript: 159-172.

Bachmann, C. \& L. Basier (1989): Mise en images d'une banlieue ordinaire: stigmatisations urbaines et stratégies de communication. - Paris: Alternatives Sociales.

Berthaut, J. (2008): La mise en image du «problème des banlieues» au prisme de la division du travail journalistique. - In: Revue Agone 40: 109-130, http://revueagone.revues.org/72 21.12.2009.

Bonelli, L. (2003): Evolutions et régulations des illégalismes populaires en France depuis le début des années 1930. - In: Cultures et Conflits 51, 3: 9-42.

Bonelli, L. (2008): La France a peur. Une histoire sociale de l'«insécurité». - Paris: La Découverte.

Brailich, A., Germes, M., Schirmel, H., Glasze, G. \& R. PÜTZ (2008): Die diskursive Konstitution von Grosswohnsiedlungen in Frankreich, Deutschland und Polen. - In: Europa Regional 16, 3: 113-128.

Brailich, A., Germes, M., Schirmel, H., Glasze, G.
\& R. PÜTZ (2010): Grosswohnsiedlungen als bedrohliche und bedrohte Orte: Sicherheitsdiskurse in Deutschland, Frankreich und Polen. - In: Soeffrner, H.-G. (Hrsg.): Unsichere Zeiten. Herausforderungen gesellschaftlicher Transformationen. - Wiesbaden: VS Verlag für Sozialwissenschaften (CD-ROM).

Coutras, J. (2003): Les peurs urbaines et l'autre sexe. - Paris: L'Harmattan.

DiKeç, M. (2006): Badlands of the Republic? Revolts, the French state, and the question of banlieues. - In: Environment \& Planning D: Society and Space 24, 2: 159-163.

DiKeç, M. (2007): Space, governmentality and the geographies of French urban policy. - In: European Urban and Regional Studies 14, 4: 277-289.

Ducrot, O. (1984): Le dire et le dit. - Paris: Éditions de Minuit.

Estèbe, P. (2004): L'usage des quartiers. Action publique et géographie dans la politique de la ville (1982-1999). - Paris: L'Harmattan.

FAYMAn, S. (1995): La politique de la ville ... et les habitants? Jeunes et femmes, passerelles entre leur milieu et l'action politique. - In: Les Annales de la recherche urbaine 68-69: 114-122.

Foucault, M. (1966): Les mots et les choses. Une archéologie des sciences humaines. - Paris: Gallimard. Foucault, M. (1969): Archéologie du savoir. - Paris: Gallimard.

Foucault, M. (2005 [1984]): Die Heterotopien. Der utopische Körper. - Frankfurt am Main: Suhrkamp Verlag.

Germes, M., Schirmel, H., Brailich, A., Glasze, G. 
\& R. PÜTZ (2010): Menaces pour l'ordre social? Une lecture géographique et politique des discours sur les grands ensembles de banlieue en Allemagne, France et Pologne. - In: Annales de Géographie (im Druck).

Glasze, G. (2009): Der Raumbegriff bei Laclau. Auf dem Weg zu einem politischen Konzept von Räumen. - In: Glasze, G. \& A. Mattissek (Hrsg.): Handbuch Diskurs und Raum. Theorien und Methoden für die Humangeographie sowie die sozial- und kulturwissenschaftliche Raumforschung. - Bielefeld: transcript: 213-218.

Glasze, G., Germes, M. \& F. Weber (2009): Krise der Vorstädte oder Krise der Gesellschaft? - In: Geographie und Schule 177: 17-25.

Glasze, G. \& A. Mattissek (2009): Die Hegemonie- und Diskurstheorie von Laclau und Mouffe. - In: Glasze, G. \& A. Mattissek (Hrsg.): Handbuch Diskurs und Raum. Theorien und Methoden für die Humangeographie sowie die sozial- und kulturwissenschaftliche Raumforschung. - Bielefeld: transcript: 153-179.

Gregory, D. (1994): Geographical imaginations. Cambridge: Blackwell.

GrEGORY, D. (1995): Imaginative Geographies. - In: Progress in Human Geography 19, 4: 447-485.

GrEGORY, D. (2004): The colonial present: Afghanistan, Palestine, Irak. - Malden: Blackwell.

Hagmann, J. (2010): Räume der Unsicherheit: Konstruktion, Emanzipation und Exklusion durch Sicherheitspolitik. - In: Geographica Helvetica 65, 3: 172180.

Hancock, C. (2008): Décoloniser les représentations: esquisse d'une géographie culturelle de nos «Autres». - In: Annales de Géographie 660/661:116-128.

HöHNE, T. (2003): Die Thematische Diskursanalyse - dargestellt am Beispiel von Schulbüchern. - In: Keller, R., Hirseland, A., Schneider, W. \& W. VieHÖVER (Hrsg.): Handbuch Sozialwissenschaftliche Diskursanalyse, Band 2: Forschungspraxis. - Opladen: Leske + Budrich: 389-419.

HusseinI, S. (2009): Die Macht der Übersetzung. Konzeptionelle Überlegungen zur Übersetzung als politische Praktik am Beispiel kulturgeographischer Forschung im arabischen Sprachraum. - http://www. soc-geogr-discuss.net/5/145/2009/sgd-5-145-2009.pdf 7.9.2009.

HÜSER, D. (2004): RAPublikanische Synthese. Eine französische Zeitgeschichte populärer Musik und politischer Kultur. - Köln: Böhlau Verlag.

JABLONKA, Y. (2008): La naissance du «casseur». Délinquance et ethnicité dans la République (1981-2006). - In: Caron, J.-C., Stora-Lamarre, A. \& J.-J. Yvorel (Hrsg.): Les âmes mal nées. Jeunesse et délinquance urbaine en France et en Europe (XIXe-XXe siècles). - Actes du colloque international, Besançon, 15, 16 et 17 novembre 2006, Besançon: Presses universitaires de Franche-Comté: 153-164.
Kalinic, A. (2008): Les types d'argumentation dans le traitement du débat sur la crise des banlieues par la télévision. - In: Argumentation \& Analyse du Discours 1: 1-13, http://aad.revues.org/index198.html 21.12.2009.

LACLAU, E. (1990): New reflections on the revolution of our time. - London, New York: Verso.

Laclau, E. \& C. Mouffe (1985): Hegemony and socialist strategy. Towards a radical democratic politics. - London, New York: Verso.

LAPEYRONNIE, D. (2005): La banlieue comme théâtre colonial, ou la fracture coloniale dans les quartiers. - In: Blanchard, P., Bancel, N. \& S. Lemaire (Hrsg.): La fracture coloniale: la société française au prisme de l'héritage colonial. - Paris: La Découverte: 209-218.

Marchart, O. (1998): Kunst, Raum und Öffentlichkeit(en). - http://eipcp.net/transversal/0102/marchart/de 8.12.2009.

Mattissek, A. (2009): Die Aussagenanalyse als Mikromethode der Diskursforschung. - In: Glasze, G. \& A. Mattissek (Hrsg.): Handbuch Diskurs und Raum. Theorien und Methoden für die Humangeographie sowie die sozial- und kulturwissenschaftliche Raumforschung. - Bielefeld: transcript: 279-291.

Rigouste, M. (2008): La guerre à l'intérieur: la militarisation du contrôle des quartiers populaires. - In: MucCHIELLI, L. (Hrsg.): La frénésie sécuritaire. Retour à l'ordre et nouveau contrôle social. - Paris: La Découverte: 88-98.

Romieux, C. (2007): Logement social et traitement de l'insécurité. - Paris: L'Harmattan.

SAID, E.W. (1978): Orientalism. - London: Routledge. Sauvadet, T. (2006): Jeunes dangereux, jeunes en danger: comprendre les violences urbaines. - Paris: Dilecta.

Schumann, A. (2005): Culture banlieue: zur Protestkultur der Migrantenkinder in Frankreich. - In: FendLER, U. (Hrsg.): Grenzenlos. Festschrift für Helmut Schwartz zum 65. Geburtstag. - Aachen: Shaker Verlag: 125-134.

Tissot, S. \& F. Poupeau (2005): La spatialisation des problèmes sociaux. - In: Actes de la recherche en sciences sociales 159: 4-9.

Tissot, S. (2007): L'Etat et les quartiers. Genèse d'une catégorie de l'action publique. - Paris: Seuil.

WÆVEer, O. \& B. BuZAN (2007): After the return to theory: the past, present and future of security studies. - In: Collins, A. (Hrsg.): Contemporary security studies. - Oxford: Oxford University Press: 383-402.

Wihtol de Wenden, C. (2007): Une histoire des banlieues: la fabrique des territoires urbains. - In: BoDYGendrot, S. \& C. Wintol de Wenden (Hrsg.): Sortir des banlieues. Pour en finir avec la tyrannie des territoires. - Paris: Autrement: 17-47.

ŽıžEK, S. (1998 [1990]): Jenseits der Diskursanalyse. In: Marchart, O. (Hrsg.): Das Undarstellbare der Politik. Zur Hegemonietheorie Ernesto Laclaus. - Wien: Turia \& Kant: 123-131. 
Zusammenfassung: Die banlieues als Gegenorte der République. Eine Diskursanalyse neuer Sicherheitspolitiken in den Vorstädten Frankreichs

Die banlieues sind Ziel spezifischer Sicherheitspolitiken und werden diskursiv als bedrohliche und fremde Orte konstituiert, wo die Regeln und Werte der République nicht gelten. In diesem Sinne dienen die banlieues als konstitutives Aussen und Gegenorte der République. Auf paradoxe Weise werden die banlieues dabei als «verlorene Territorien» konstituiert, die «eigentlich» zur République gehören und deshalb zurückerobert werden müssen. Die Wiederaneignung wird durch eine Differenzierung innerhalb der Anderen legitimiert: die gefährlichen Anderen müssen durch die République zurückgedrängt werden, um die gefährdeten Anderen integrieren zu können.

Schlüsselwörter: Diskursforschung, Stadtforschung, Polizei, nationale Identität, Frankreich

\section{Abstract: The banlieues as the counterplaces of the République. A discourse analysis of current security policies in French suburbs}

The French banlieues are currently subject to particular security policies and are depicted in discourse as threatening and foreign places, i.e. places where the norms and the rules of the République do not apply. In this sense the banlieues serve as a constitutive outside and counterplace of the République. Paradoxically, they are at the same time described as «lost territories» that should be integrated back into the republican society. This «recapturing» is legitimised by a differentiation of the others between «the dangerous others» who have to be repressed and «the others at risk» who need to be rescued and integrated.

Keywords: discourse theory, urban studies, police, national identity, France
Résumé: Les banlieues comme contre-lieux de la République. Une analyse discursive des nouvelles politiques de sécurité dans les périphéries françaises

Cette contribution montre comment, dans les discours politiques français, les banlieues font l'objet de politiques de sécurité spécifiques et sont constituées comme des lieux menaçants et étrangers, où les règles et les valeurs de la République ne sont pas respectées. En ce sens, elles représentent le lieu de l'Autre, le «dehors constitutif», le «contre-lieu» de la République. Elles sont paradoxalement aussi considérées comme «territoires perdus» qui appartiennent en fait à la République et qui doivent pour cette raison être reconquis. Cette réappropriation est légitimée grâce à une différenciation discursive au sein des Autres, entre ceux qui sont désignés comme dangereux et ceux qui sont considérés comme en danger. Les premiers doivent être repoussés afin de pouvoir intégrer les seconds.

Mots-clés: théorie des discours, géographie urbaine, police, identité nationale, France

Dr. Mélina Germes, Prof. Dr. Georg Glasze, Institut für Geographie der Universität Erlangen-Nürnberg, Kochstrasse 4/4, D-91054 Erlangen, Deutschland. e-mail:

mgermes@geographie.uni-erlangen.de gglasze@geographie.uni-erlangen.de

Manuskripteingang/received/manuscrit reçu le 23.1.2010

Annahme zum Druck/accepted for publication/accepté pour publication: 6.9 .2010 